\title{
Особенности транспорта электронов в двумерных квантовых сверхрешетках с неассоциативным законом дисперсии
}

\author{
() М.Л. Орлов ${ }^{1}$, Л.К. Орлов ${ }^{2,3}$ \\ ${ }^{1}$ Нижегородский институт управления при президенте Российской Федерации, филиал РАНХиГС, \\ 603950 Нижний Новгород, Россия \\ ${ }^{2}$ Нижегородский государственный технический университет им. Р.Е. Алексеева, \\ 603950 Нижний Новгород, Россия \\ ${ }^{3}$ Институт фризики микроструктур Российской академии наук, \\ 603950 Нижний Новгород, Россия \\ E-mail: orlov@ipm.sci-nnov.ru
}

Поступила в Редакцию 9 марта 2020 г.

В окончательной редакции 14 сентября 2020 г.

Принята к публикации 26 октября 2020 г.

\begin{abstract}
Для двумерной квантовой сверхрешетки с негармоническим законом дисперсии электронов изучены анизотропия проводимости и вид вольт-амперной характеристики для различных относительно осей сверхрешетки направлений тока и прикладываемого к структуре поля. Для разных режимов протекания тока обсуждаются анизотропия характеристик и условия появления многозначных вольт-амперных характеристик. Показано, что отклонение закона дисперсии электронов в двумерной квантовой сверхрешетке от гармонического в сильном постоянном электрическом поле оказывает заметное влияние на вид вольт-амперных характеристик двумерной квантовой сверхрешетки. Появление в сильном поле нескольких всплесков тока связано как с перемешиванием линий тока для ортогональных относительно прикладываемого поля направлений, так и с транспортом электронов по уровням штарковских лестниц, формируемых в разных долинах мини-зоны.
\end{abstract}

Ключевые слова: двумерные сверхрешетки, неассоциативный закон дисперсии, анизотропия, постоянное электрическое поле, вольт-амперная характеристика, штарковские состояния.

DOI: $10.21883 /$ FTP.2021.03.50602.9393

\section{1. Введение}

Интерес к двумерным (2Д) гетеро- и нанокомпозициям, в частности квантовым сверхрешеткам (СР), в значительной степени связан с прогрессом, наблюдаемым в развитии нанотехнологий, и с успехами в разработке нанотранзисторов, квантовых каскадных лазеров и лазеров на квантовых точках. Стремительное развитие технологии биполярных и полевых гетеротранзисторов с туннельной эмиссией электронов в наноразмерную базовую (канальную) область структуры делает изучение особенностей характеристик поверхностных наноструктурированных периодических композиций актуальным уже на современном этапе. Для формирования двумерных квантовых сверхрешеток (2ДСР) сегодня чаще всего предлагают использовать массивы связанных туннелированием квантовых точек и нанопор $[1,2]$. Интерес представляют также естественные сверхструктуры, реализуемые, в частности, на базе гетерокомпозиций со слоями нанокристаллического карбида кремния [3]. В настоящее время в литературе широко обсуждаются возможности наблюдения в нанокристаллитах карбида кремния эффектов пространственного ограничения [4]. Активно изучаются также свойства различных аллотропных форм кристаллов карбида кремния, характеризуемых присутствием в объеме структуры естественной сверхрешетки [3,5]. Очевидно, что разработка подобных систем значительно расширяет диапазон свойств уже реализованных на базе одномерных сверхрешеток квантовых электронных устройств и позволяет с новых позиций рассмотреть особенности характеристик нанокристаллических материалов.

Среди явлений, обсуждаемых применительно к периодическим полупроводниковым гетероструктурам, особое место занимает эффект блоховской генерации, экспериментальные исследования которого в одномерных квантовых сверхрешетках GaAs/AlGaAs были начаты в 90-е годы [6,7]. Несмотря на трудности, стоящие на пути изготовления блоховского генератора, он продолжает привлекать внимание многочисленных исследователей благодаря низкому уровню критических полей генерации излучения в нем и возможностям использования его в диапазоне сверхвысоких частот [8]. Интерес к двумерным квантовым сверхрешеткам связан с тем, что в определенных условиях, согласно [9], 2ДСР характеризуются бо́льшими по сравнению с одномерными периодическими структурами временами жизни блоховских осцилляций. Это позволяет повысить мощность генерации и эффективность преобразования высокочастотного излучения в 2ДСР, делая привлекательным их использование на практике.

Теоретические исследования электронных характеристик двумерных квантовых сверхрешеток в настоящее время, однако, представлены весьма ограниченным числом работ с преимущественной ориентацией на простейший гармонический закон дисперсии электронов в 
СР с квадратной решеткой $[1,2,9,10]$. С другой стороны, эффект блоховской генерации связан с транспортом электронов по узкой мини-зоне и может зависеть от характера закона дисперсии электронов, реализуемого в ней в направлении прикладываемого электрического поля. Диагностика мини-зонного спектра и соответственно выявление механизма наблюдаемой в СР генерации, однако, весьма затруднительны, так как в сильном электрическом поле закон дисперсии электронов в СР трансформируется в систему дискретных уровней, образующих в общем случае систему штарковских лестниц $[2,11]$. В связи с этим изучение особенностей отклика системы на внешнее возмущение, обусловленных влиянием исходного (в отсутствие электрического поля) вида закона дисперсии электронов на ванье-штарковское квантование спектра электронов в мини-зонах СР, представляется достаточно важной задачей.

В настоящей работе в различных режимах протекания тока по структуре изучается вид вольт-амперных характеристик (BAX) двумерной СР. В присутствии постоянного электрического поля $\mathbf{E}$ проведен анализ особенностей анизотропии транспортных свойств латеральных квантовых СР с более сложным, чем простой гармонический, законом дисперсии электронов. Обсуждаются анизотропия проводимости и механизмы, обусловливающие проявление на ВАХ различного рода особенностей. Интерес к СР со сложным законом дисперсии электронов связан, с одной стороны, с расширением круга полупроводниковых материалов, используемых в настоящее время для изготовления периодических гетероструктур. С другой стороны, на транспортные свойства носителей заряда в 2ДСР значительное влияние в сильном постоянном электрическом поле оказывает анизотропия рассеяния электронов, обусловливая перемешивание квантовых состояний для различных кристаллографических направлений. В связи с этим в системе могут проявиться дополнительные механизмы, оказывающие влияние на эффект отрицательной проводимости, проявляющийся как на низких, так и на высоких частотах.

\section{2. Транспорт электронов в двумерных квантовых сверхрешетках в сильном постоянном электрическом поле}

Далее для различных режимов протекания тока изучаются особенности транспортных характеристик латеральных квантовых 2ДСР. В отличие от предшествующих работ $[1,12,13]$, в которых рассматривалась простая квадратная решетка с гармоническим законом дисперсии электронов, в настоящей работе обсуждаются электрические характеристики 2ДСР с более сложной зависимостью энергии носителей заряда от квазиимпульса. Запишем закон дисперсии электронов в двумерной мини-зоне в следующем виде:

$$
\begin{aligned}
\varepsilon(\mathbf{k})= & \varepsilon\left(k_{3}\right)+\varepsilon\left(\mathbf{k}_{\perp}\right)=\varepsilon\left(k_{3}\right)+\Delta_{1}\left\{1-\left[\Delta_{11} \cos \left(k_{1} d_{1}\right)\right.\right. \\
& \left.\left.+\Delta_{12} \cos \left(k_{2} d_{2}\right)\right] /\left(\Delta_{11}+\Delta_{12}\right)\right\} \\
& +\Delta_{2}\left\{1-\delta_{0} \cos \left(k_{1} d_{1}\right) \cos \left(k_{2} d_{2}\right)\right\},
\end{aligned}
$$

где $\varepsilon(\mathbf{k})$ и $\mathbf{k}$ - энергия и волновой вектор электрона, $k_{i}$ - его компоненты, $\Delta_{1(2)}, \Delta_{11}, \Delta_{12}, \delta_{0}= \pm 1-$ параметры энергетической зоны двумерной квантовой сверхрешетки. Введение диссоциативного $\left(\sim \Delta_{2}\right)$ члена приводит к усложнению закона дисперсии, обусловливая возможность появления в двумерной зоне Бриллюэна дополнительных боковых экстремумов. Положение последних весьма чувствительно как к выбору значений параметров, так и к выбору направления (угол $\theta$ вектора $\mathbf{k}$ относительно главной оси $x_{1}$ ). В общем случае характер зависимости $\varepsilon\left(\mathbf{k}_{\perp}\right)$ определяется выбором материалов гетеропары. Представление зависимости $\varepsilon\left(k_{3}\right)$ в виде $\varepsilon\left(k_{3}\right)=\hbar^{2} k_{3}^{2} / 2 m_{3}^{*}$ может быть использовано, например, для 2ДСР, состоящих из периодического набора расположенных вертикально и связанных туннелированием одномерных квантовых нитей, формируемых в нанопористом фотонном кристалле. Другим примером могут служить текстурированные поликристаллы (например, структуры $3 C$ - $\mathrm{SiC} / \mathrm{Si}(100))$ с наноразмерным масштабом зерен, растущие колоннами в вертикальном плоскости роста направлении [3]. В двумерном массиве квантовых точек для $\varepsilon\left(k_{3}\right)$ естественно предположить $\varepsilon\left(k_{3}\right)=\varepsilon_{03}=$ const, где $\varepsilon_{03}$ соответствует нижнему уровню размерного квантования в направлении, перпендикулярном плоскости 2ДСР. В общем случае будем полагать $d_{1} \neq d_{2}$, что является характерным для обсуждаемых в литературе массивов квантовых точек, располагающихся вдоль формируемых на поверхности кристалла параллельных направляющих канавок.

На первом этапе для выявления особенностей, обусловливаемых спецификой закона дисперсии электронов, аналогично работам $[1,12]$ проведем расчет вольт-амперной характеристики (BAX) двумерной квантовой сверхрешетки, используя уравнение Больцмана с интегралом столкновений в приближении постоянного времени релаксации:

$$
\begin{aligned}
\partial f / \partial t+ & (e / \hbar) E_{1}(t) \partial f / \partial k_{1}+(e / \hbar) E_{2}(t) \partial f / \partial k_{2} \\
= & -\left(f-f^{0}\right) / \tau
\end{aligned}
$$

где $f(k, t)$ и $f^{0}(k)$ - неравновесная, возмущенная полем, и равновесная функции распределения электронов. Приближение, выбранное для интеграла столкновений, является вполне приемлемым, чтобы выявить основные особенности электрических характеристик, связанные с видом используемого в нижней мини-зоне закона дисперсии электронов, и одновременно учесть эффект перепутывания состояний для разных направлений. Далее наряду с 2ДСР, характеризуемой квадратной элементарной ячейкой $\left(d_{1}=d_{2}=d\right)$, естественной, например, для 
нанокристаллических текстурированных пленок $3 C$ - $\mathrm{SiC}$, осаждаемых на поверхности $\mathrm{Si}(100)$ [3], будем рассматривать также характеристики сверхрешеток с прямоугольной ячейкой $\left(d_{1} \neq d_{2}\right)$. Вариантом последней, как уже указывалось выше, являются линейчатые структуры, состоящие из периодической последовательности квантовых точек с периодом $d_{1}$, формируемых вдоль естественных либо искусственных направляющих, разделенных диэлектрическими прослойками.

В одно-мини-зонном приближении выражение для плотности тока записывается в виде

$$
\mathbf{j}=\left(e / 4 \pi^{3} \hbar\right) \int_{\Omega} f(\mathbf{k})(\partial \varepsilon / \partial \mathbf{k}) \partial \mathbf{k} .
$$

Используя условие периодичности, представим функцию распределения в виде ряда Фурье:

$$
\begin{gathered}
f\left(k_{1}, k_{2}, t\right)=\sum_{\nu, \mu=-\infty}^{\infty} F_{v \mu} \Phi_{\nu \mu}(t) \exp \left\{i\left(v k_{1} d_{1}+\mu k_{2} d_{2}\right)\right\} \\
F_{v \mu}=\left(1 / 8 \pi^{3}\right) \int_{-\infty}^{\infty} \partial k_{3} \int_{0}^{2 \pi} \partial\left(k_{1} d_{1}\right) \int_{0}^{2 \pi} \partial\left(k_{2} d_{2}\right) f^{0}(\mathbf{k}) \\
\times \exp \left\{-i\left(v k_{1} d_{1}+\mu k_{2} d_{2}\right)\right\} .
\end{gathered}
$$

Выражение для $F_{v \mu}$ зависит от размерности модели и выбора равновесной функции распределения. Для распределения Больцмана и закона дисперсии (1) соответственно получаем:

$$
\begin{aligned}
F_{\nu \mu} & =F_{0} \operatorname{Re} \int_{0}^{2 \pi} \partial x_{1} \int_{0}^{2 \pi} \partial x_{2} \exp \left(i v x_{1}+i \mu x_{2}\right) \\
& \times \exp \left\{Y_{11} \cos \left(x_{1}\right)+Y_{12} \cos \left(x_{2}\right)+Y_{20} \cos \left(x_{1}\right) \cos \left(x_{2}\right)\right\},
\end{aligned}
$$

где

$$
\begin{gathered}
x_{1,2}=k_{1(2)} d_{1(2)}, D_{10}=\Delta_{1} /\left\{\left(\Delta_{11}+\Delta_{12}\right)\right\}, D_{11}=D_{10} \Delta_{11}, \\
D_{12}=D_{10} \Delta_{12}, D_{20}=\delta_{0} \Delta_{2}, Y_{i j}=D_{i j} / k_{\mathrm{B}} T, \\
F_{0}=\left\{\left(2 \pi m_{3} k_{\mathrm{B}} T\right)^{1 / 2} / 16 \pi^{3} \hbar\right\} \exp \left\{\left(\mu_{\mathrm{F}}-\Delta_{1}-\Delta_{2}\right) / k_{\mathrm{B}} T\right\} .
\end{gathered}
$$

При выводе (6) полагалось, что направления векторов $\mathbf{k}_{1}$ и $\mathbf{k}_{2}$ совпадают с направлениями главных осей прямоугольной решетки: $\mathbf{k}_{1}\left\|[100], \mathbf{k}_{2}\right\|$ [010]. Функция $\Phi_{\nu \mu}(t)$ находится из решения уравнения (2):

$$
\begin{aligned}
\Phi_{\nu \mu}= & \exp \left\{-\left(t-t_{0}\right) / \tau\right\}+\tau^{-1} \int_{t_{0}}^{t} \exp \left\{\left(t^{\prime}-t\right) / \tau-i(e / \hbar)\right. \\
& \left.\times \int_{t^{\prime}}^{t}\left[v E_{1}\left(t^{\prime \prime}\right) d_{1}+\mu E_{2}\left(t^{\prime \prime}\right) d_{2}\right] \partial t^{\prime \prime}\right\} \partial t^{\prime} .
\end{aligned}
$$

В случае постоянного электрического поля $\mathbf{E}=$ const c компонентами $E_{1,2}$ вдоль осей $x_{1,2}$ при начальном моменте времени включения поля $t_{0}=-\infty$ имеем

$$
\Phi_{\nu \mu}=\left\{1-i \tau\left[\nu \Omega_{1}+\mu \Omega_{2}\right]\right\} /\left\{1+\tau^{2}\left[\nu \Omega_{1}+\mu \Omega_{2}\right]^{2}\right\},
$$

где $\quad \Omega_{1}=\left(e d_{1} / \hbar\right) E_{1}, \quad E_{1}=E \cos \psi, \quad \Omega_{2}=\left(e d_{2} / \hbar\right) E_{2}$, $E_{2}=E \sin \psi, \quad \Omega \tau=E / E_{0}, \quad E_{0}=\hbar / e d_{1} \tau, \quad \psi \quad$ - угол между направлением поля и осью $x_{1}$. Подставляя (1), (4), (6), (8) в уравнение (3), окончательно получим выражения для токовых компонент вдоль главных осей 2ДСР с законом дисперсии (1). Соответствующие выражения для компонент плотности тока $j_{1}, j_{2}$ в направлении главных осей 2ДСР принимают вид

$$
\begin{aligned}
j_{1}= & j_{0}\left(E / E_{0}\right) \cos \psi\left\{D_{11} F_{10} /\left[1+\left(E / E_{0}\right)^{2} \cos ^{2} \psi\right]\right. \\
+ & F_{11} D_{20}\left\{1+\left(E / E_{0}\right)^{2}\left[\cos ^{2} \psi-\chi \sin ^{2} \psi\right]\right\} /\left\{1+2\left(E / E_{0}\right)^{2}\right. \\
\times & {\left.\left.\left[\cos ^{2} \psi+\chi \sin ^{2} \psi\right]+\left(E / E_{0}\right)^{4}\left[\cos ^{2} \psi-\chi \sin ^{2} \psi\right]^{2}\right\}\right\}, } \\
j_{2}= & j_{0}\left(E / E_{0}\right) \sin \psi\left\{D_{12} F_{01} /\left[1+\chi\left(E / E_{0}\right)^{2} \sin ^{2} \psi\right]\right. \\
+ & F_{11} D_{20}\left\{1-\left(E / E_{0}\right)^{2}\left[\cos ^{2} \psi-\chi \sin ^{2} \psi\right]\right\} /\left\{1+2\left(E / E_{0}\right)^{2}\right. \\
& {\left.\left.\left[\cos ^{2} \psi+\chi \sin ^{2} \psi\right]+\left(E / E_{0}\right)^{4}\left[\cos ^{2} \psi-\chi \sin ^{2} \psi\right]^{2}\right\}\right\}, }
\end{aligned}
$$

где $j_{0}=e F_{0} / 2 \pi \hbar d_{2}, \chi=\left(d_{2} / d_{1}\right)^{2}$. Если ввести параметр $d_{\psi}$, соответствующий периоду СР в направлении прикладываемого поля $\mathbf{E}\left(d_{1}=d_{\psi} \cos \psi, E_{\psi}=\hbar / e d_{\psi} \tau\right)$, тогда компоненты тока (9), (10) можно переписать в виде

$$
\begin{aligned}
j_{1}= & j_{0}\left(E / E_{\psi}\right)\left\{D_{11} F_{10} /\left[1+\left(E / E_{\psi}\right)^{2}\right]+F_{11} D_{20}\left[1+\left(E / E_{\psi}\right)^{2}\right.\right. \\
& \left.\times\left(1-\chi \operatorname{tg}^{2} \psi\right)\right] /\left[1+2\left(E / E_{\psi}\right)^{2}\left(1+\chi \operatorname{tg}^{2} \psi\right)\right. \\
& \left.\left.+\left(E / E_{\psi}\right)^{4}\left(1-\chi \operatorname{tg}^{2} \psi\right)^{2}\right]\right\}, \\
j_{2}= & j_{0}\left(E / E_{\psi}\right) \operatorname{tg} \psi\left\{D_{12} F_{01} /\left[1+\chi\left(E / E_{\psi}\right)^{2} \operatorname{tg}^{2} \psi\right]\right. \\
& +F_{11} D_{20}\left[1-\left(E / E_{\psi}\right)^{2}\left(1-\chi \operatorname{tg}^{2} \psi\right)\right] /\left[1+2\left(E / E_{\psi}\right)^{2}\right. \\
& \left.\left.\times\left(1+\chi \operatorname{tg}^{2} \psi\right)+\left(E / E_{\psi}\right)^{4}\left(1-\chi \operatorname{tg}^{2} \psi\right)^{2}\right]\right\} .
\end{aligned}
$$

В формулах (9), (11) первые слагаемые в фигурных скобках описывают вольт-амперную характеристику одномерной (вдоль оси $x_{1}$ ) квантовой СР. Для двумерной квантовой СР в отсутствие неаддитивного слагаемого в законе дисперсии (1), т.е. при $D_{20}=0$, выражения для плотностей токов $(9),(10)$ были получены и исследованы в работе [1]. Здесь же впервые была изучена анизотропия электрических характеристик квантовой сверхрешетки. В частности, было показано, что перемешивание электронных состояний в продольном и поперечном полю направлениях приводит к несовпадению направлений поля и тока, протекающего по структуре. Степень анизотропии возрастала с ростом величины прикладываемого электрического поля. 


\section{3. Особенности вольт-амперной характеристики двумерной квантовой сверхрешетки с неаддитивным законом дисперсии при заданном направлении поля}

Предварительный анализ ВАХ, проведенный в [12] для простейшей квадратной решетки $\left(D_{11}=D_{12}\right)$, показал, что присутствие неаддитивного слагаемого $\left(\Delta_{2} \neq 0\right)$ в законе дисперсии (1) сказывается на вольт-амперной характеристике, смещая максимум ВАХ в область более высоких или более низких значений поля относительно критического поля нелинейности $E_{0}$. В сильных полях на ВАХ 2ДСР с неаддитивным законом дисперсии в принципе возможно появление дополнительного второго максимума. Далее мы более детально рассмотрим свойства статической проводимости и особенности ВАХ, обусловленные неаддитивностью закона дисперсии электронов в 2ДСР как с одинаковыми, так и с разными значениями периодов СР $d_{1}, d_{2}$ вдоль главных осей симметрии.

В общем случае величина тока, протекающего по структуре, определяется выражением $j=\left(j_{1}^{2}+j_{2}^{2}\right)^{1 / 2}$, соотношение $j_{2} / j_{1}=\operatorname{tg} \varphi$ задает направление тока относительно оси $x_{1}$, определяемое углом $\varphi$. Как уже отмечалось в работах $[1,12]$, основной особенностью 2ДСР является несовпадение направлений поля (угол $\psi$ ) и протекающего по структуре тока (угол $\varphi$ ). Для двумерной решетки исключение составляют оси высокой симметрии, т.е. углы $\psi=\varphi=0, \pi / 4, \pi / 2$ (при $d_{1}=d_{2}$ ) и $\psi=\varphi=0, \pi / 2$ (при $\left.d_{1} \neq d_{2}\right)$. Несовпадение углов $\psi$ и $\varphi$ связано с асимметрией функции распределения в интеграле столкновений, возникающей вследствие перепутывания квазиимпульсов $k_{1}$ и $k_{2}$ при разогреве электронного газа. С ростом прикладываемого электрического поля асимметрия возрастает. При этом наблюдаемый эффект проявляется даже в структурах с чисто гармоническим законом дисперсии $\left(\Delta_{2}=0\right)$ в условиях изотропного времени релаксации $(\tau=$ const $)$ [1]. Наблюдаемое расхождение углов $\psi$ и $\varphi$ тем больше, чем больше отклонение выбранного направления поля от осей высокой симметрии и чем больше величина прикладываемого электрического поля.

\section{1. Проводимость электронов в квадратной двумерной сверхрешетке}

Для квадратной решетки с параметрами $\Delta_{1} \gg \Delta_{2}$ максимальное значение тока достигается для углов $(\psi, \varphi)$, лежащих в направлении диагонали $(\psi, \varphi \approx \pi / 4)$ при $\Omega \tau \approx \sqrt{2}$ :

$$
j \approx j_{0}\left(D_{11} F_{10}+D_{12} F_{01}\right)(\Omega \tau / \sqrt{2}) /\left[1+(\Omega \tau)^{2} / 2\right] .
$$

Для решетки с параметрами $\Delta_{1} \ll \Delta_{2}$, как и для одномерной $\mathrm{CP}$, максимальная величина тока достигается для углов $(\psi, \varphi)$, близких к нулю при $\Omega \tau=1$ :

$$
j \approx j_{0} F_{11} D_{2} \Omega \tau /\left\{1+(\Omega \tau)^{2}\right\} .
$$

В более общем случае характерные зависимости угла $\varphi$ и компонент протекающего по структуре тока $\mathbf{j}$ от направления и величины прикладываемого поля $\mathbf{E}$ для квадратной СР представлены для ряда значений параметров на рис. 1. Для квадратной СР для всех значений параметров в области высоких полей, $E>E_{\psi}$, наблюдается монотонный спад тока с ростом электрического поля.

Присутствие в законе дисперсии (1) неаддитивного слагаемого $\left(\Delta_{2} \neq 0\right)$ при отклонении направления прикладываемого к структуре электрического поля в сторону от основных осей симметрии $(\psi=0, \pi / 4)$ приводит к изменению вида вольт-амперной характеристики и,
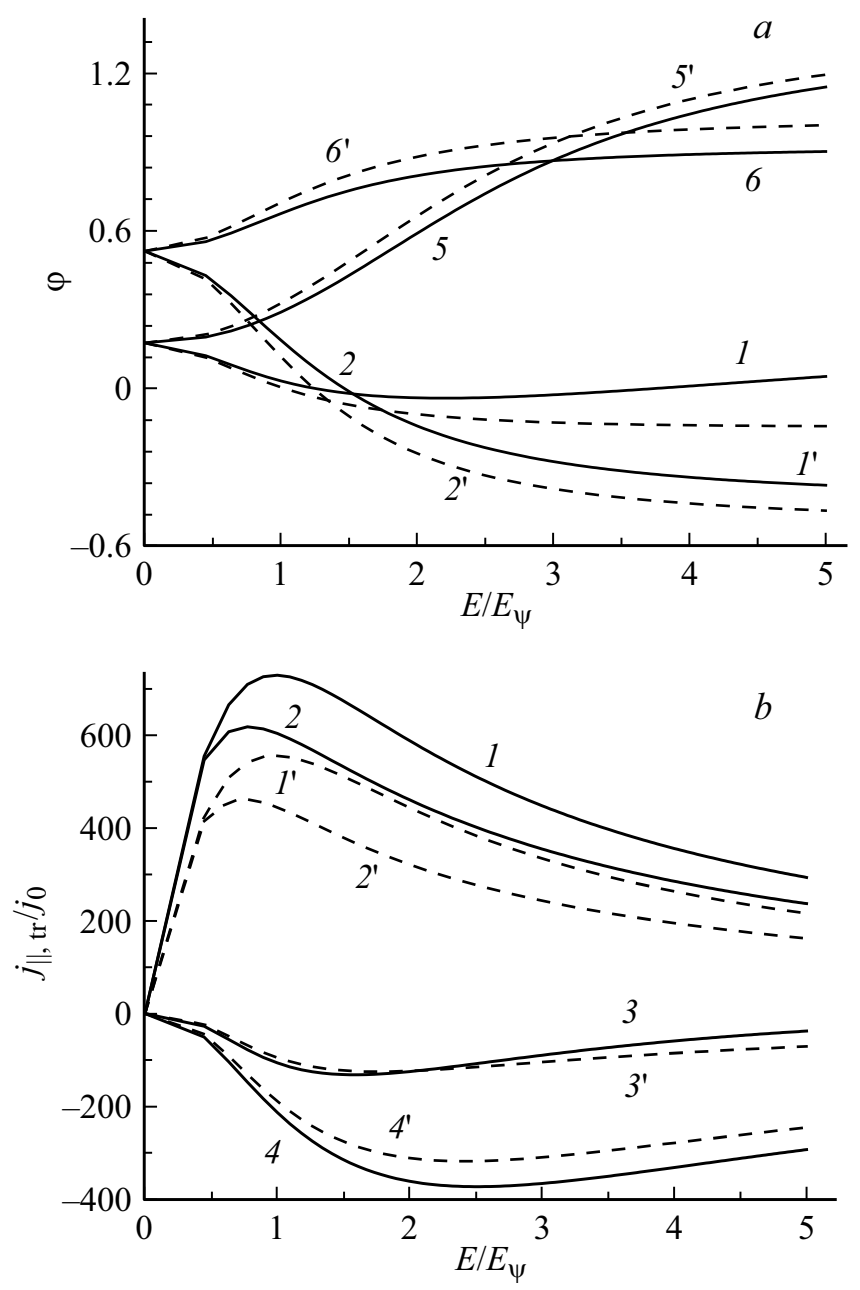

Рис. 1. Зависимости от прикладываемого поля $E$ угла наклона линий тока $\varphi(a)$ и величин $(b)$ параллельной, $j_{\|}\left(1,1^{\prime}, 2,2^{\prime}\right)$, и перпендикулярной направлению поля, $j_{\mathrm{tr}}\left(3,3^{\prime}, 4,4^{\prime}\right)$, составляющих тока $\mathbf{j}$ для углов $\psi=\pi / 18\left(1,1^{\prime}, 3,3^{\prime}, 5,5^{\prime}\right)$, $\pi / 6\left(2,2^{\prime}, 4,4^{\prime}, 6,6^{\prime}\right)$. Для параметров двумерной квадратной СР принималось: $d_{2}=d_{1}, \Delta_{1}=5$ мэВ, $\Delta_{2}=1\left(5,5^{\prime}, 6,6^{\prime}\right)$, 20 мэВ $\left(1-4,1^{\prime}-4^{\prime}\right), \Delta_{11}=\Delta_{12}=1, \delta_{0}=1 \quad(1-6$, сплошная линия), -1 ( $1^{\prime}-6^{\prime}$, штриховая линия); $k_{\mathrm{B}} T=7$ мэВ. 


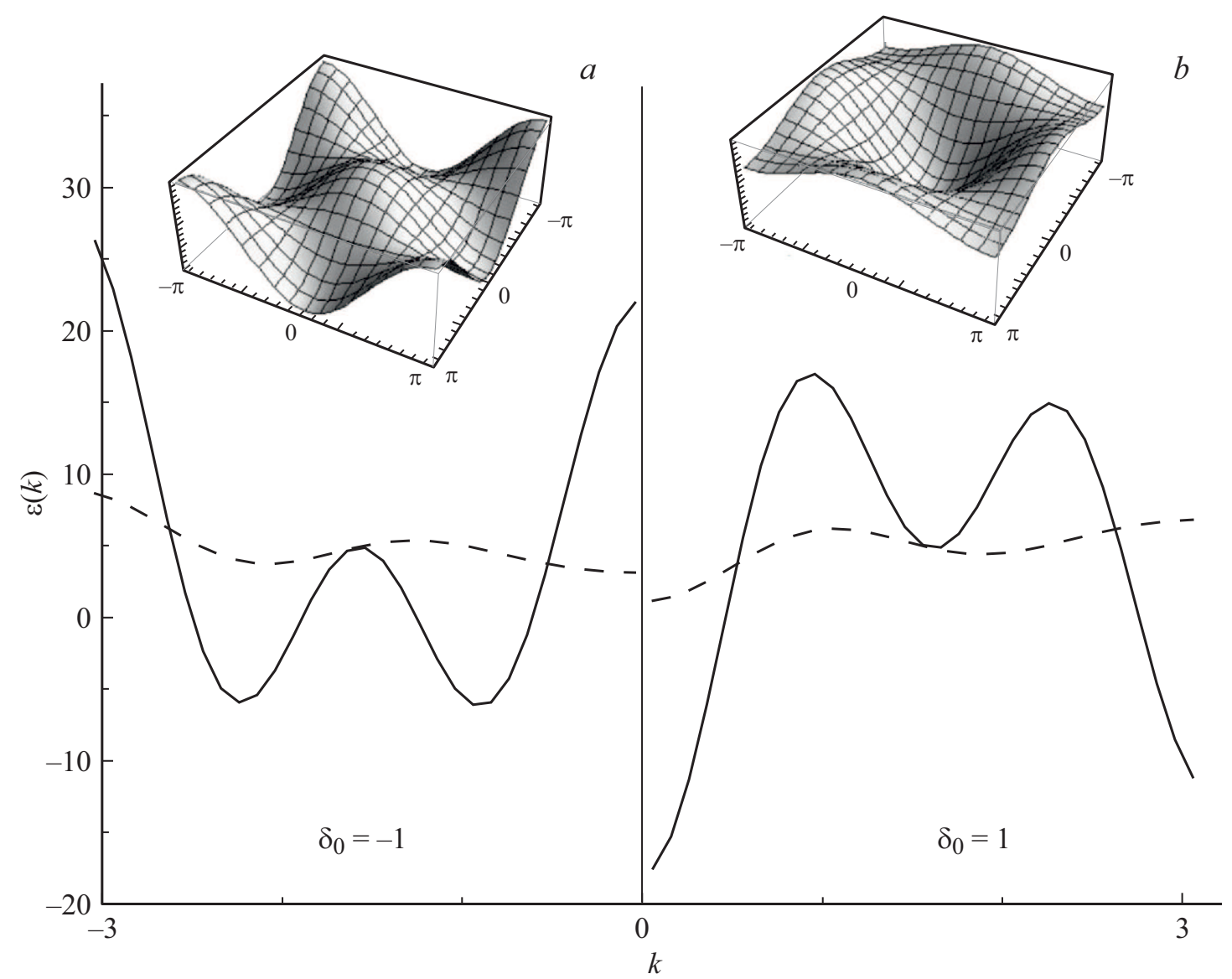

Рис. 2. Общий вид закона дисперсии $\varepsilon(\mathbf{k})$ и его вид в направлении под углом $\theta=\pi / 10$ для значений параметров $\Delta_{1}=5$ мэВ, $\Delta_{11}=\Delta_{12}=1, \Delta_{2}=1$ (штриховая линия), 20 мэВ (сплошная линия), $\delta_{0}=-1(a), 1(b) ; k_{\mathrm{B}} T=7$ мэВ. Здесь $\varepsilon-$ в мэВ, $k=k_{1,2} d_{1,2}-$ безразмерный параметр.

в частности, к появлению поперечной составляющей тока. Появление данной токовой компоненты возможно, если на торцах системы присутствуют эффективные механизмы стока носителей заряда, т.е. имеет место сильная поверхностная рекомбинация. Тогда заряды на боковой поверхности не накапливаются и направление приложенного поля не меняется. При этом, как следует из рис. $1, a$, величина угла $\varphi$ будет зависеть как от величины приложенного поля (с ростом поля разница углов $\varphi$ и $\psi$ нарастает), так и от вклада, определяемого величиной $\left(\Delta_{2}\right)$ и знаком $\left(\delta_{0}\right)$, диссоциативного слагаемого в закон дисперсии электронов. Поперечная токовая компонента при этом с ростом поля ведет себя подобно продольной компоненте, но с максимумом тока, сдвинутым в область более сильных полей. Наличие в обеих компонентах тока участков с отрицательной дифференциальной проводимостью указывает на возможность усиления высокочастотных сигналов, поляризованных как в продольном, так и в поперечном направлениях относительно направления прикладываемого постоянного электрического поля. Уменьшение вклада в законе дисперсии диссоциативного слагаемого приводит к изменению знака поперечной составляющей тока (рис. $1, a)$, что может быть использовано для диагностики закона дисперсии электронов в СР. Расчеты, проводимые для квантовой СР как с узкими, так и с широкими (относительно $k_{\mathrm{B}} T$ ) разрешенными минизонами, показывают в области $\Omega \tau>1$ зависимость дифференциальной проводимости от параметров, входящих в закон дисперсии электронов. Таким образом, даже в квантовом пределе сохраняется связь между током, текущим по дискретным уровням штарковской лестницы, и видом исходного (в отсутствие поля) закона дисперсии электронов в мини-зоне квантовой сверхрешетки.

\section{2. Проводимость электронов в двумерной сверхрешетке с прямоугольной решеткой}

Несколько иная ситуация наблюдается в прямоугольной квантовой СР $\left(d_{1} \neq d_{2}\right)$. Зависимость $\varepsilon(k)$ в этом случае характеризуется наличием боковых экстремумов, положение и глубина которых определяются величиной и знаком параметра $D_{20}$, а также выбором угла $\theta$ (рис. 2 ). Смена знака параметра $\delta_{0}$ приводит к инверсии центральной долины, что может оказаться принципиальным 
для периодических гетероструктур с перекрывающимися в соседних слоях состояниями валентной зоны и зоны проводимости.

На рис. 3 для разных значений величины диссоциативного слагаемого $\Delta_{2}$ в законе дисперсии электронов приведены зависимости от $E$ компонент тока в направлениях вдоль, $j_{\|}$, и поперек, $j_{\mathrm{tr}}$, направления прикладываемого электрического поля при фиксированном угле $\psi$. При расчетах нами учитывалось влияние на вид BAX амплитуд гармоник $F_{I J}$, входящих в выражения для токов и меняющихся при изменении значений параметров в довольно широких пределах. Сопоставление кривых на рис. 2 и 3 позволяет отчетливо проследить наличие связи между характером закона дисперсии в мини-зоне, выбором угла приложения поля и видом BAX CP.

Из приведенных рис. 2,3 видно, что изменение вида закона дисперсии с ростом параметра $\Delta_{2}$ оказывает заметное влияние на транспортные свойства электронов в сверхрешетке. При этом инверсия центральной долины при простой смене знака параметра $\delta_{0}$ не сказывается принципиальным образом на качественном виде ВАХ СР. Наблюдаемая на $\mathrm{BAX} j_{\|}(E)$ при $\Omega \tau \gg 1$ область отрицательной дифференциальной проводимости (ОДП) (рис. 2), связанная с механизмом блоховских осцилляций, заметно сужается как с увеличением величины диссоциативного слагаемого, пропорционального $\Delta_{2}$, так и при изменении угла $\psi$ (рис. 2,a). В сильном поле, $E>E_{\psi}$, с ростом параметра $\Delta_{2}$ плотность тока нарастает, приводя к появлению на токовой характеристике дополнительного широкого максимума, что, вероятнее всего, является следствием перераспределения электронов между соседними долинами. В сильном поле ВАХ 2ДСР становится подобной характеристике одномерной СР, в которой имеет место эффективное перераспределение электронов между соседними мини-зонами вследствие туннелирования между мини-зонами $[11,14,15]$. Можно предположить, что в 2ДСР со сложным многодолинным законом дисперсии спектр электронов в сильном поле трансформируется не в одну, а в систему штарковских лестниц, переходы между которыми и приводят к наблюдаемому изменению вида ВАХ. Чтобы уменьшить вклад электронов в боковых долинах в формирование ВАХ 2ДСР, достаточно изменить угол прикладываемого поля относительно главных осей 2ДСР (рис. 2,a). В качестве механизма, обусловливающего появление на ВАХ 2ДСР второго максимума, можно предположить также возможное перепутывание вследствие анизотропии интеграла столкновений состояний в перпендикулярных полю направлениях. Данный эффект приводит к смешиванию продольной и поперечной проводимости системы, аналогично тому, как это имеет место в измерениях квантового эффекта Холла методом Ван дер Пау на квадратном пробнике [16].

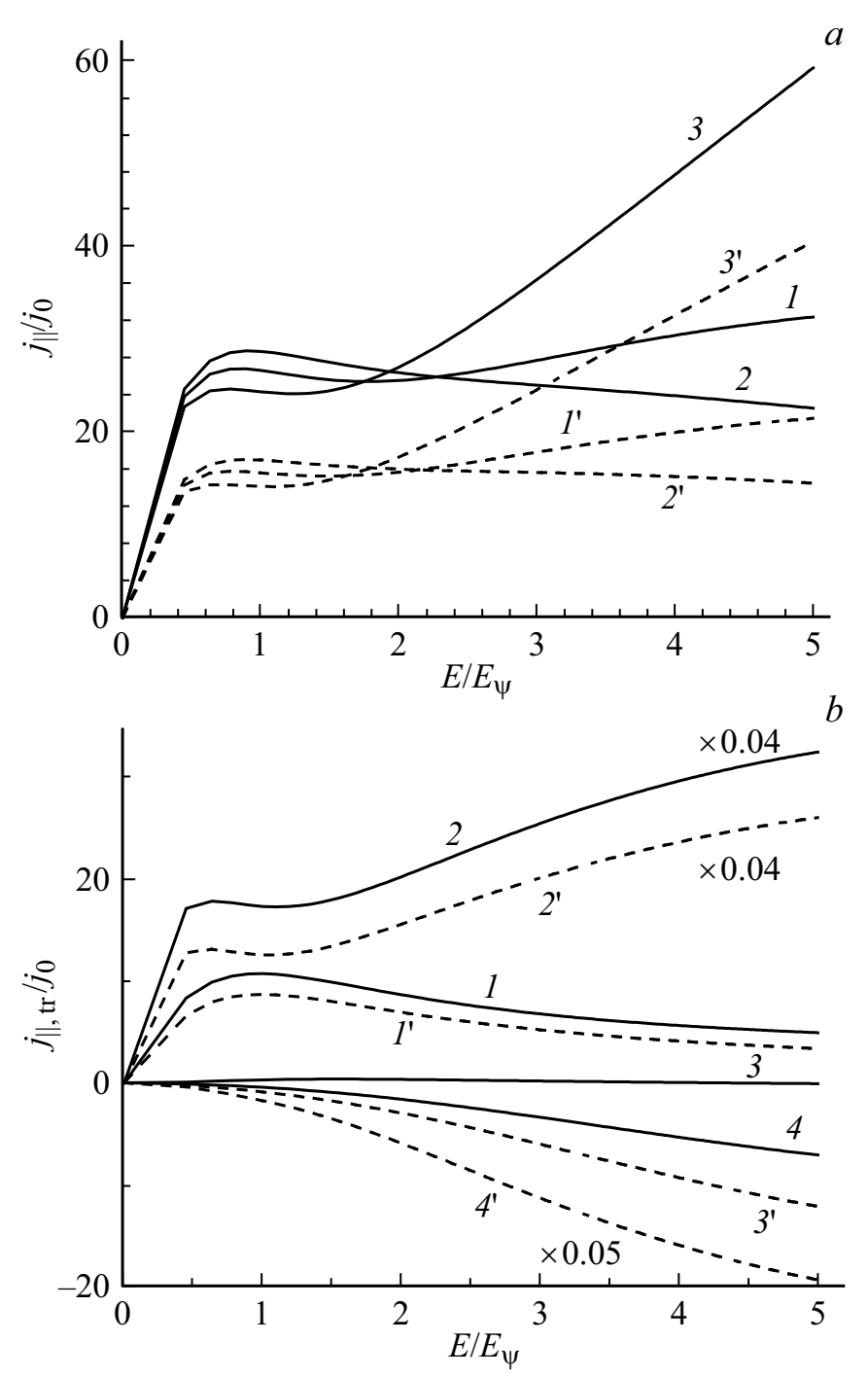

Рис. 3. Зависимости от поля $E$ компонент тока для ряда значений параметра $\Delta_{2}$ и при некоторых фиксированных углах $\psi$ наклона поля. $a-$ компонента $j_{\|}, \psi=\pi / 10\left(1,1^{\prime}\right)$, $\pi / 11\left(2,2^{\prime}\right), \pi / 9\left(3,3^{\prime}\right), \Delta_{2}=5$ мэВ, $\delta_{0}=1(1-3$, сплошная линия), -1 ( $1^{\prime}-3^{\prime}$, штриховая линия). $b-$ параллельная направлению поля компонента $j_{\|}\left(1,1^{\prime}, 2,2^{\prime}\right)$ и перпендикулярная компонента $j_{\operatorname{tr}}\left(3,3^{\prime}, 4,4^{\prime}\right), \psi=\pi / 10, \Delta_{2}=1\left(1,1^{\prime}, 3,3^{\prime}\right)$, 20 мэВ $\left(2,2^{\prime}, 4,4^{\prime}\right), \delta_{0}=1$ ( $1-4$, сплошная линия), $-1\left(1^{\prime}-4^{\prime}\right.$, штриховая линия). Для остальных параметров принимались значения: $d_{2} / d_{1}=2.7, \Delta_{1}=5$ мэВ, $\Delta_{11}=\Delta_{12}=1, k_{\mathrm{B}} T=7$ мэВ.

Эффект затухания блоховских осцилляций, обусловленный перепутыванием штарковских состояний в направлениях вдоль и поперек направления текущего по структуре тока, впервые обсуждался в работах [2,9]. Очевидно, что более детальную информацию о структуре квантовых состояний в сверхрешетке в постоянном электрическом поле можно получить, только проводя одновременно анализ высокочастотных резонансных характеристик системы, подобно тому, как это было сделано, например, для одномерной квантовой СР [17]. 


\section{4. Особенности вольт-амперной характеристики двумерной квантовой сверхрешетки в режиме фиксируемого направления тока}

Выше мы рассмотрели ситуацию, соответствующую выбору заданного направления поля относительно осей 2ДСР. Этот режим можно реализовать, используя конфигурацию поверхностного слот-диода, располагая, например, планарную сверхрешетку между истоковым и стоковым контактами полевого транзистора. В реальном эксперименте, однако, может быть реализован также случай отсутствия эффективного рекомбинационного стока на боковых торцах структуры. Тогда вследствие накопления заряда на боковых гранях поле будет отклоняться от своего первоначального направления и возникнет поперечная направлению тока компонента электрического поля [1]. В связи с этим представляется необходимым рассмотреть ситуацию фиксированного направления тока $(\varphi=$ const $)$. Далее будем полагать, что направление тока задано и угол $\varphi$ равен углу между векторами $\mathbf{j}$ и $\mathbf{x}_{1}$. Этот режим реализуется, например, на образцах, выполненных в конфигурации холловского мостика. Направление поля в образце, определяемое углом $\psi$, в этом случае будет зави-

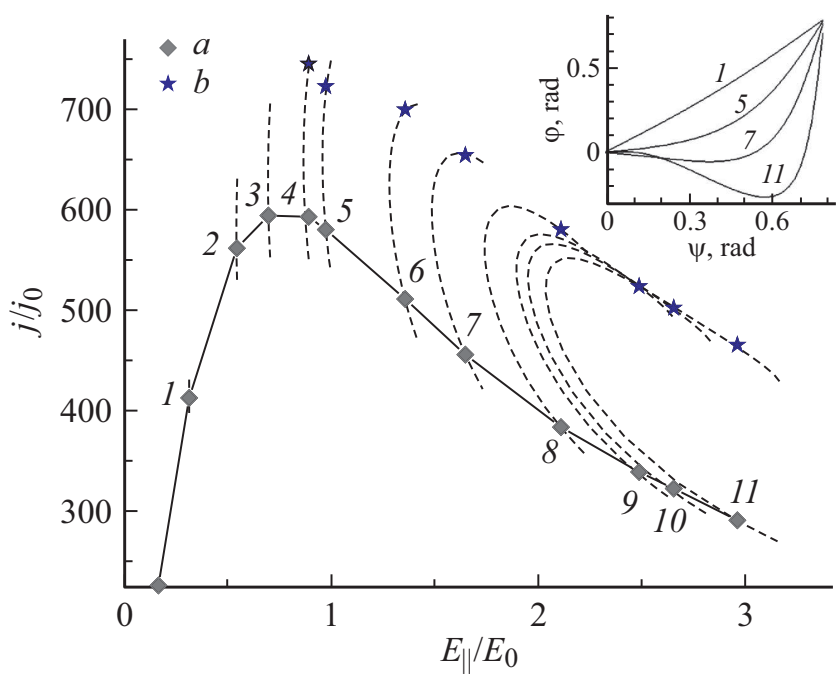

Рис. 4. Зависимости величины тока $j$ в структуре от параллельной току компоненты поля $E_{\|}$при фиксированном угле $\varphi=0.4$ рад для квадратной $\mathrm{CP}$ с параметрами $d_{2} / d_{1}=1, \quad \Delta_{1}=5$ мэВ $, \quad \Delta_{11}=\Delta_{12}=1, \quad \Delta_{2}=20$ мэВ, $\delta_{0}=1$ при $k_{\mathrm{B}} T=7$ мэВ и величинах прикладываемого поля $\left(E / E_{0}\right)^{2}=0.1 \quad(1), 0.3 \quad(2), 0.5 \quad(3), 0.8 \quad(4), 1$ (5), 2 (6), 3 (7), 5 (8), 7 (9), 8 (10), 10 (11). На вставке - для ряда значений параметра $(E / E)^{2}$ показаны зависимости угла наклона линий тока $(\varphi)$ от угла наклона линий поля $(\psi)$. Штриховые кривые - зависимости $j(E)$ при фиксированном направлении поля (фиксированном угле $\psi)$. Точки $(a-$ основное решение, $b$ - дополнительное решение) соответствуют токам на линиях тока (штриховые кривые) для фиксированных значений компоненты поля $E_{\|}$. сеть как от величины поля, так и от других параметров системы.

Для вычисления ВАХ 2ДСР при фиксированном направлении тока можно использовать соотношения (11), (12), устанавливающие для заданной величины прикладываемого электрического поля зависимость между компонентой тока $j_{\|}$и углом $\psi$. Далее, используя взаимосвязь между углами $\varphi$ и $\psi$ (см. вставку на рис. 4), определяемую при постоянном значении поля соотношением $\varphi=\operatorname{arctg}\left(j_{2} / j_{1}\right)$, нетрудно установить зависимость величины полного тока $j$ от угла $\varphi$. Затем, ориентируясь на полученные зависимости, для фиксированных значений поля $E$ и угла $\varphi$ подбираем значения тока $j$ и продольной (вдоль направления тока) компоненты поля $E_{\|}$(точки на рис. 4 ).

С целью проведения более точного анализа рассмотрим решения уравнений токопереноса (11), (12), фиксируя направление тока, т.е. полагая $\varphi=$ const. В этом случае, согласно проведенному в работах $[4,8]$ предварительному анализу, в системе будет появляться, аналогично эффекту Холла, компонента поля, направленная поперек пропускаемому через образец току. Пусть направление тока, текущего по структуре, отклонено от одной из главных осей СР (ось $x_{1}$ ) на угол $\varphi$, лежащий в интервале углов между 0 и $\pi / 4$. В этом случае продольная $\left(\mathbf{E}_{\|} \| \mathbf{j}\right)$ и поперечная $\left(\mathbf{E}_{\perp} \perp \mathbf{j}\right)$ компоненты электрического поля $\mathbf{E}$ и тока $\mathbf{j}$ связаны с их проекциями на главные оси СР соотношениями

$$
\begin{gathered}
j_{1}=j \cos \varphi, \quad j_{2}=j \sin \varphi \\
E_{\|}=E \cos (\psi-\varphi), \quad E_{\perp}=E \sin (\psi-\varphi) .
\end{gathered}
$$

Связь между направлением (угол $\psi)$ и величиной поля $E$ при заданном угле $\varphi$ можно установить, используя условие равенства нулю тангенциальной составляющей тока: $\operatorname{tg} \varphi=j_{2} / j_{1}=$ const. Искомая зависимость $j_{\|}\left(E_{\|}\right)$ находится путем подстановки найденного соотношения между $E$ и $\psi$ в выражение для плотности тока $j=j_{\|}=\left(j_{1}^{2}+j_{2}^{2}\right)^{1 / 2}$, где $j_{1,2}$ определяются соотношениями (11), (12).

Рассмотрим прежде всего простейший случай $\varphi=0$. В этом случае из условия $j_{2}=0$ сразу находим одно решение $\psi^{(1)}=0$, соответствующее совпадающим по направлению току и полю. Выражение для плотности тока в этом случае принимает вид

$$
j^{(1)}=j_{1}=j_{01} \Omega^{(1)}\left(D_{11} F_{10}+F_{11} D_{20}\right) /\left\{1+\left(\tau \Omega^{(1)}\right)^{2}\right\} .
$$

Однако равенство нулю компоненты тока $j_{2}$ допускает также возможность существования и другого решения. Наиболее простое решение имеет место при переходе к СР с преимущественно диссоциативным законом дисперсии. В частности, при $\Delta_{12}=0$ связь между направлением $\psi^{(2)}$ и величиной $E^{(2)}$ поля имеет вид: 
$\psi^{(2)}= \pm 1 / 2 \arccos \left[1 /\left(\tau \Omega^{(2)}\right)^{2}\right]$, откуда следует:

$$
\begin{gathered}
j^{(2)}=j_{01} \Omega_{\|}^{(2)}\left(D_{11} F_{10}+F_{11} D_{20}\right) / 2 ; \\
\Omega_{\|}^{(2)}=\Omega^{(2)} \cos \left\{0.5 \operatorname{arcos}\left[1 /\left(\tau \Omega^{(2)}\right)^{2}\right]\right\} .
\end{gathered}
$$

Связь между углом $\psi$ (для $0<\psi<\pi / 2$ ) и блоховской частотой $\Omega$, а также зависимости тока (при $\varphi=0$ ) от параллельной току компоненты поля $E_{\|}$, описываемые соотношениями (16), (17), показаны на рис. 5, $a$ (кривые 1).

При $\Delta_{12} \neq 0$ и $\varphi=0$ в СР с квадратной зоной Бриллюэна уравнение, связывающее направление поля (угол $\psi$ ) с его величиной $E / E_{0}$, имеет вид квадратного уравнения относительно как переменной $\Phi=\cos 2 \psi$, так и переменной $W=\left(E / E_{0}\right)^{2}$ :

$$
\begin{aligned}
& D_{12} F_{01}\left[1+2 W+W^{2} \cos ^{2} 2 \psi\right] \\
& +F_{11} D_{20}(1-W \cos 2 \psi)[1+W / 2-(W / 2) \cos 2 \psi]=0 .
\end{aligned}
$$

Решая уравнение (18), находим: $\psi= \pm 0.5 \arccos (\Phi)$, где $\Phi=\left\{-b \pm\left[b^{2}-4 a c\right]^{1 / 2}\right\} / 2 a, a=W^{2}\left(D_{12} F_{01}+D_{20} F_{11} / 2\right) ;$ $b=-F_{11} D_{20}(3+W) W / 2 ; \quad c=D_{12} F_{01}(1+2 W)+F_{11} D_{20}$ $\times(1+W / 2)$. Подставляя полученное решение в (11), нетрудно рассчитать полевые зависимости тока $j_{1}\left(E_{1}\right)$ и поперечной компоненты поля $E_{2}\left(E_{1}\right)$ (рис. $5, a$, кривые 2).

При $\Delta_{12} \neq 0$ и $\varphi=0$ в СР с прямоугольной решеткой $\left(d_{2} / d_{1} \neq 1\right)$ уравнение, связывающее направление поля (угол $\psi$ ) и величину поля $E_{\|} / E_{\psi}$, также имеет вид квадратного уравнения относительно величин $Z=\chi \operatorname{tg}^{2} \psi$ и $W=\left(E / E_{\psi}\right)^{2}$ :

$$
\begin{aligned}
A_{1}[1+2 W(1+Z) & \left.+W^{2}(1-Z)^{2}\right] \\
& +A_{2}[1-W+W Z][1+W Z]=0,
\end{aligned}
$$

где $\chi=\left(d_{2} / d_{1}\right)^{2}, A_{1}=\Delta_{12} F_{01}, A_{2}=\Delta_{2} F_{11}$. Решая уравнение (19), находим: $\psi= \pm \operatorname{arctg}(Z / \chi)$, где $Z=$ $=\left\{-b \pm\left[b^{2}-4 a c\right]^{1 / 2}\right\} / 2 a, \quad a=W^{2}\left(A_{1}+A_{2}\right), \quad b=-W^{2}$ $\times\left(2 A_{1}+A_{2}\right)+2 W\left(A_{1}+A_{2}\right), \quad c=A_{1}(1+W)^{2}+A_{2}(1-W)$, и, подставляя полученное решение в (11), нетрудно найти зависимости $j_{1}\left(E_{1}\right)$ и $E_{2}\left(E_{1}\right)$ (рис. $5, a$, кривые 3,4 ).

Для произвольного угла $\varphi$ задача усложняется, так как требует решения уравнения 3-го порядка, устанавливающего связь между параметрами $W$ и $\psi$. В общем случае можно использовать также процедуру, рассмотренную выше при построении кривых, представленных на рис. 4. Наиболее просто полученное уравнение решается только для двух частных случаев, соответствующих равенству нулю значений одного из параметров: $\Delta_{2}$ либо $\Delta_{1}$. Эти условия соответствуют чисто ассоциативному либо чисто диссоциативному законам дисперсии электронов. Связь между величиной и направлением прикладываемого поля в этих случаях при $d_{1}=d_{2}=d$ принимает вид

$$
\begin{aligned}
\tau^{2} \Omega^{2}= & \left\{D_{11} F_{10} \cos \psi-\sin \psi D_{12} F_{01}\right\} / \\
& \left\{D_{12} F_{01} \cos \psi-D_{11} F_{10} \sin \psi\right\} \sin \psi \cos \psi
\end{aligned}
$$
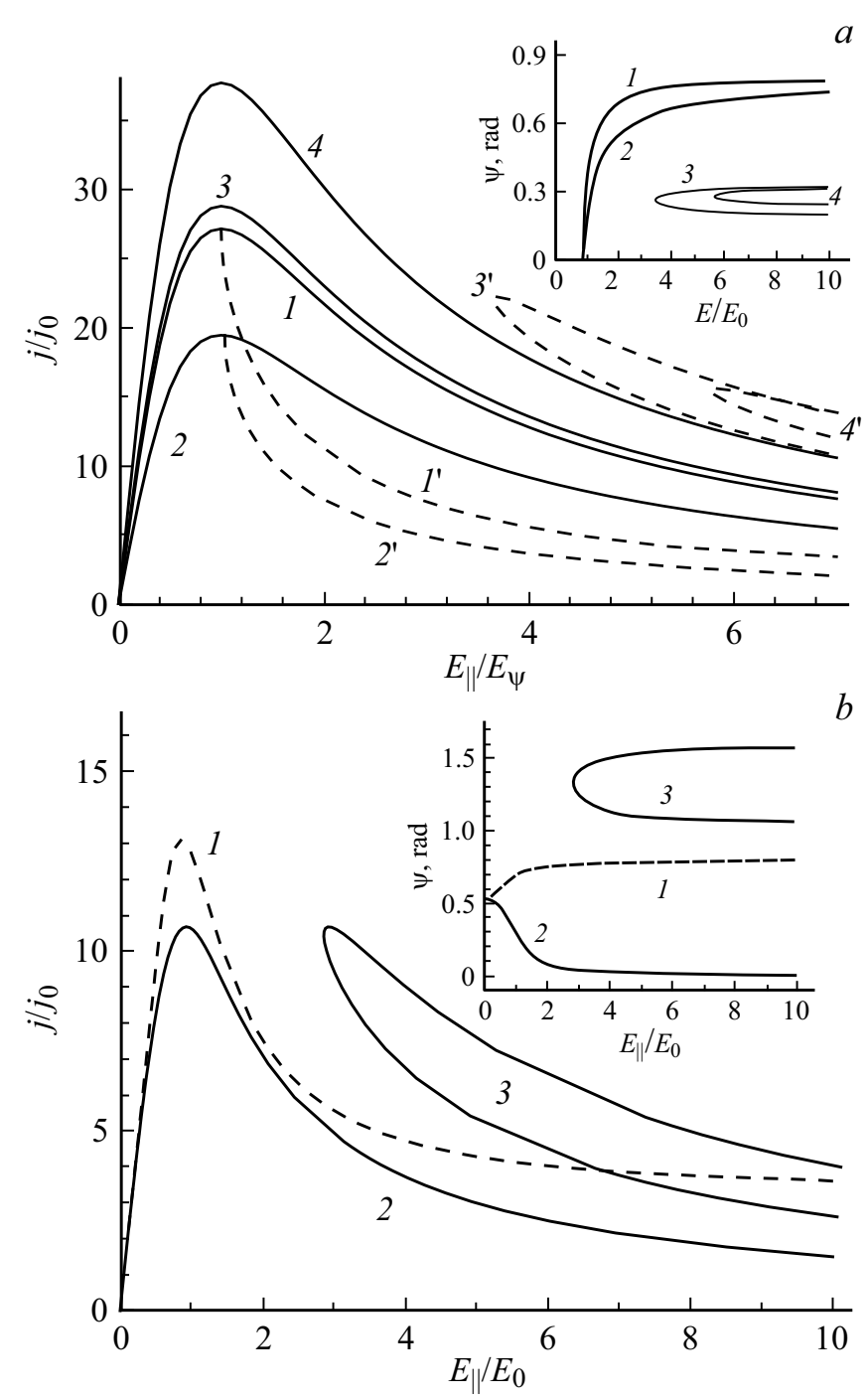

Рис. 5. Зависимости величины тока в структуре $j$ от продольной компоненты поля $E_{\|}$для фиксированных значений угла $\varphi=0(a), \pi / 6(b)$ и значений параметров $\delta_{0}=1, \Delta_{11}=1$, $k_{\mathrm{B}} T=7$ мэВ. $a-\Delta_{1}=5(1,3,4), 1$ мэВ $(2) ; \Delta_{12}=0\left(1,1^{\prime}\right)$, $1\left(2,2^{\prime}, 3,3^{\prime}\right), 3\left(4,4^{\prime}\right) ; \Delta_{2}=20(1), 7$ (2), 5 мэВ $(3,4) . b-$ $\Delta_{12}=1 ; 1-\Delta_{1}=0, \Delta_{2}=5 \mathrm{мэB} ;(2,3)-\Delta_{1}=5 \mathrm{мэB}, \Delta_{2}=0$, основное (2) и дополнительное (3) решения. $a$ : $1,2-$ квадратная СР $\left(d_{2} / d_{1}=1\right), 3,4-$ прямоугольная СР $\left(d_{2} / d_{1}=3 / 1\right)$. $b$ : квадратная СР $\left(d_{2} / d_{1}=1\right)$. На вставках - соответствующие зависимости угла $\psi$ наклона линий поля от величины прикладываемого поля $E$.

при $\Delta_{2}=0$ и

$$
\tau^{2} \Omega^{2}=(\operatorname{tg} \psi-\operatorname{tg} \varphi) /(\operatorname{tg} \psi+\operatorname{tg} \varphi) \cos 2 \psi
$$

при $\Delta_{1}=0$. Характерный вид зависимостей угла наклона $\psi$ линий поля и величины протекающего тока $j=j_{\|}$ от величины поля $E_{\|}$вдоль линий тока при заданном угле $\varphi=\pi / 6$ и значениях параметров $\Delta_{11}=\Delta_{12}=1$, $\delta_{0}=1, k_{\mathrm{B}} T=7,\left(\Delta_{1}=0, \Delta_{2}=5 \mathrm{мэB}\right)-$ штриховая линии $(1),\left(\Delta_{1}=5\right.$ мэВ, $\left.\Delta_{2}=0\right)-$ сплошные линии ( 2 - основное решение, 3 - дополнительное решение), 
представлен на рис. $5, b$. Смена знака параметра $\delta_{0}$ оказывает незначительное влияние на вид представленных на рис. 5 кривых.

Распад токовой характеристики на несколько ветвей, впервые подмеченный в работе [1], характеризуется появлением поперечной относительно направления тока компоненты электрического поля, что может сказываться на поведении высокочастотных характеристик. Несмотря на существование нескольких ветвей токовой характеристики, система, однако, будет оставаться устойчивой благодаря наличию балансира, в качестве которого выступает поперечное электрическое поле. Отклонение тока приводит к мгновенному появлению компоненты поля $E_{\perp}$, противодействующей появлению поперечной составляющей тока и восстановлению равновесия в системе, соответствующего требованию постоянства направления протекающего по структуре тока (в рассматриваемом случае условию $\varphi=0$ ). Вследствие этого скачки между разными ветвями тока будут носить кратковременный характер, а направление поля при этом будет испытывать колебания в обе стороны от заданного направления тока с углами $\pm \psi$.

\section{5. Заключение}

Проведен анализ особенностей анизотропии и транспортных свойств латеральных двумерных квантовых СР со сложным многодолинным законом дисперсии электронов в нижней мини-зоне 2ДСР. Анизотропия спектра квантовой сверхрешетки в сильном постоянном электрическом поле через интеграл столкновений приводит к перемешиванию квантовых уровней штарковских лестниц в направлениях вдоль и поперек линий протекания тока. Перемешивание штарковских уровней заметным образом сказывается на виде ВАХ, приводя для случая квадратной СР к замедлению спада тока в полях выше критического, $E>E_{0}$. Для прямоугольной $\mathrm{CP}$ в ряде случаев возможен рост тока и появление на ВАХ СР в сильных полях при определенных углах протекания тока дополнительного широкого максимума. Эффект подобен влиянию туннельного пробоя между уровнями штарковских лестниц соседних мини-зон, наблюдаемому на ВАХ одномерных СР. Можно предположить, что в 2ДСР данная особенность ВАХ СР связана с перебросом электронов между уровнями формируемых в зоне Бриллюэна серии штарковских лестниц от групп носителей, локализованных в соседних долинах. Показано, что исходный вид закона дисперсии электронов в мини-зоне оказывает заметное влияние на формирование штарковских уровней и характер блоховских осцилляций, что проявляется в поведении отклика системы на внешнее поле.

\section{Благодарности}

Работа посвящена памяти проф. Ю.А. Романова, которым были инициированы как постановка задачи, так и начало ее решения. Работа выполнена при поддержке Российского фонда фундаментальных исследований, проект 18-42-520062.

\section{Конфликт интересов}

Авторы заявляют об отсутствии конфликта интересов.

\section{Список литературы}

[1] Ю.А. Романов, Е.В. Демидов. ФТП, 31 (3), 308 (1997).

[2] И.А. Дмитриев, Р.А. Сурис. ФТП, 35 (2), 219 (2001).

[3] Л.К. Орлов, В.И. Вдовин, Н.Л. Ивина. ФТТ, 61 (7), 1322 (2019).

[4] J.Y. Fan, X.L. Wu, P.K. Chu. Progr. Mater. Sci., 51, 983 (2006).

[5] В.И. Санкин, А.А. Лепнева. ФТП, 34, 831 (2000).

[6] C. Waschke, H.G. Roskos. R. Schwedler, K. Leo, H. Kurz, K. Koler. Phys. Rev. Lett., 70 (21) 3319 (1993).

[7] E. Schomburg, R. Scheuerer, S. Brandl, K.F. Renk, D. Pavel'ev, Yu. Kochurinov, V. Ustinov, A. Zhukov, A. Kovsh, P.S. Kop'ev. Electron. Lett., 35 (17), 1491 (1999).

[8] Yu.A. Romanov, L.G. Mourokh, N.J.M. Horing. J. Appl. Phys., 93, 4696 (2003).

[9] И.А. Дмитриев, Р.А. Сурис. ФТП, 36 (12), 1460 (2002).

[10] С.Ю. Глазов, С.В. Крючков. ФТП, 35 (4), 456 (2001).

[11] Л.К. Орлов. Изв. вузов СССР. Радиофизика, 32 (12), 1550 (1989).

[12] M.L. Orlov, Yu.A. Romanov, L.K. Orlov. Microelectronics J., 36, 396 (2005).

[13] M.L. Orlov, Yu.A. Romanov, L.K. Orlov. Proc. First Int. Workshop on Semicond. Nanocrystals (Budapest, Hungary, 2005) v. 2, p. 325.

[14] Л.К. Орлов, Ю.А. Романов. ФТП, 19 (10), 1877 (1985).

[15] Л.К. Орлов. Радиотехника и электроника, $33(6), 1278$ (1988).

[16] O.A. Mironov, O.A. Kuznetsov, L.K. Orlov, R.A. Rubtsova, A.L. Chernov, S.V. Chistyakov, M. Oszwaldovskii, B.A. Aronzon, N.K. Chumakov. Superlatt. Microstr., 10 (4), 467 (1991).

[17] Л.К. Орлов, Ю.А. Романов. Изв. вузов СССР. Радиофизика, 32 (3), 282 (1989).

Редактор Л.В. Шаронова 


\section{Features of electron transport in two-dimensional quantum superlattices with the non-associative dispersion low}

M.L. Orlov ${ }^{1}$, L.K. Orlov ${ }^{2,3}$

${ }^{1}$ Russian Presidential Academy of National Economy and Public Administration, 603950 Nizhny Novgorod, Russia

${ }^{2}$ Nizhny Novgorod State Technical University

n. a. R.E. Alekseev,

603950 Nizhny Novgorod, Russia

${ }^{3}$ Institute for Physics of Microstructures,

Russian Academy of Sciences,

603950 Nizhny Novgorod, Russia

Abstract The anisotropy of conductivity and the form of current-voltage $(I-V)$ characteristics for different directions (relative to the axes of the superlattice) of current and field applied to the structure are studied for a two-dimensional quantum superlattice with an electron dispersion law more complex than simple harmonic. The anisotropy of the characteristics and the conditions for the appearance of multivalued ones are discussed for different current flow regimes. It is shown that deviation of the electron dispersion law in a two-dimensional quantum superlattice from harmonic in a strong constant electric field has a noticeable effect on the current-voltage characteristic form of two-dimensional quantum superlattice. The appearance of several peaks of the current in a strong field is due to mixing of the Stark electron levels for orthogonal directions and the Stark electron levels of different valleys. 